\title{
An assessment of the macrophage electrophoretic mobility test (MEM) in cancer diagnosis
}

\author{
E. H. CROZIER, MARY E. HOLlingER, BERTHA E. WOODEND, AND J. H. \\ ROBERTSON
}

The Laboratories, Belfast City Hospital, Belfast BT97AD, N. Ireland

SYNOPSIS The results of an assessment of the macrophage electrophoretic mobility test in cancer $\overline{0}$ diagnosis are described. We found that the test did not reliably distinguish between a group of $18 \mathrm{~N}$ patients with benign disorders and a group of 25 with malignant disease.

There have been conflicting reports on the value of the macrophage electrophoretic mobility (MEM) test in the diagnosis of cancer. Field and Caspary (1970) found that the peripheral blood lymphocytes of patients with cancer were sensitized to a basic protein derived from human brain (encephalitogenic factor). They showed that, when incubated with this antigen, these sensitized lymphocytes released a macrophage slowing factor which could be detected by its effect on the electrophoretic mobility of guinea-pig macrophages. These findings were confirmed by Pritchard et al $(1972,1973)$ and by Goldstone et al (1973), both of whom used a modification of the original method. However, Lewkonia et al (1974), using both the original and the modified techniques, were unable to show that the MEM test reliably discriminated between patients with benign and malignant disease. Because a test for cancer is clearly of great importance, we report here our own experience with the MEM test.

\section{Patients and methods}

Peripheral blood lymphocytes from 18 control subjects with non-malignant disease and from 25 with malignant disease were used in the investigation. Of the latter group, 23 had a carcinoma, one a choriocarcinoma, and one Hodgkin's disease. The methods used were those described by Pritchard et al (1973). Lymphocyte suspension in TC199 was prepared from venous blood using a Ficoll-Triosil method and incubated $\left(23^{\circ} \mathrm{C}\right)$ with encephalitogenic factor (EF) prepared from human brain, as described by Caspary and Field (1971). Guinea-pig macrophages were obtained by injecting Hartley albino

Received for publication 3 December 1975 female guinea-pigs (250-500 g) intraperitoneally with $\vec{\complement}$ warm sterile liquid paraffin. The suspension of macrophages $\left(10^{7} / \mathrm{ml}\right)$ was irradiated to a dose of $\vec{\varphi}$ $200 \mathrm{rad}$ from a cobalt source to inactivate the guinea-pig lymphocytes and incubated $\left(37^{\circ} \mathrm{C}\right)$ with the supernatant fluid obtained from the incubation mixture of human lymphocytes and EF. Control tubes using human lymphocytes, but without EF, were also prepared. All solutions used in the test $\frac{\circ}{\varnothing}$ were maintained at $\mathrm{pH} 7 \cdot 2$. Electrophoretic mobility of the macrophages was determined using a Zeiss cytopherometer with an applied current of $8 \mathrm{~mA}$. 3 Macrophages sharply in focus in the stationary plane and filling approximately one square of the eyepiece grid were timed in both directions across a distance? of $16 \mu \mathrm{m}$ using a digital electronic stopwatch. Cells: whose times did not differ by more than $10 \%$ in each direction of travel were recorded until a series of $10 \AA$ such cell timings were measured. Determination of $₹$ the degree of macrophage slowing was made byo comparing the results from test and control tubes according to the formula outlined by Caspary ando Field (1971). On every occasion the cytopherometer operator was unaware whether the patient being. tested suffered from a benign or malignant disorder. $N$

\section{Results}

Like others who have described the MEM test, we found that satisfactory operation of the cytopherometer was achieved only after much experience 0 with the instrument. Even then the test was time-o consuming and the performance of the cytopherometer capricious.

The results are illustrated in the figure, which $\frac{D}{\sigma}$ shows the mean percentage change in macrophage mobility produced by the addition of antigen to theo 


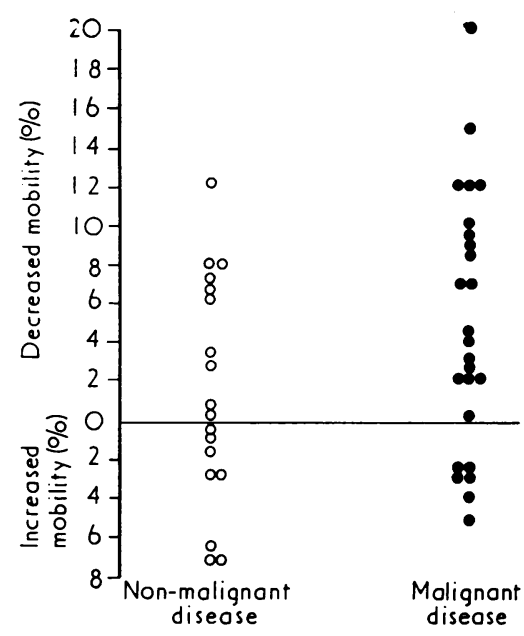

Figure Results of macrophage electrophoretic mobility test on 43 patients

test in each case. A positive test in a patient with malignant disease should be indicated by a decrease in macrophage mobility brought about by the release of macrophage slowing factor from sensitized lymphocytes. Macrophage mobility in the 18 patients with non-malignant disorders displayed a range of $6 \%$ increase in mobility to a decrease of $12 \%$. In eight of these patients mobility was increased, and in 10 it was decreased. In the 25 patients with malignant disease, mobility ranged from an increase of $5 \%$ to a decrease of $20 \%$. In six of this group mobility was increased by the antigen, and in the remaining 19 it was decreased. We were not able to find any correlation between the stage of the carcinoma and the results of the test.

\section{Discussion}

In our experience, the MEM test using the Zeiss cytopherometer is of no value as a test for malignancy. There is some suggestion in our results that patients with malignant disease do indeed show decreased macrophage mobility compared to those with benign disorders. However, there is a marked overlap of the two groups. Like other investigators we found that considerable experience with the cytopherometer was required to obtain consistent and satisfactory results. Problems with cell drifting and directional bias in migration times could be overcome only with patience and care when using the instrument.

It seems unlikely, therefore, that the results can be attributed to faulty technique as they were obtained only after operation of the instrument was considered satisfactory and they were no different towards the end of the series than at the outset. Furthermore, the overlap between the benign and malignant groups which we obtained is strikingly similar to that reported by Lewkonia et al.

During the course of this work Preece and Light (1974) described the successful use of the MEM test in cancer diagnosis using differing apparatus and techniques. This investigation was mainly carried out using capillary cell electrophoresis apparatus. However, the importance of this modification is uncertain as in a number of instances where a comparison was made with the Zeiss cytopherometer they obtained the same result on both types of instrument.

This work was supported by a grant from the Cancer Research Campaign. We wish to thank the Northern Ireland Radiotherapy Service for their valuable assistance. Our thanks are also due to Dr J. A. V. Pritchard for advice and to Miss B. Maguire, who prepared the manuscript.

\section{References}

Caspary, E. A., and Field, E. J. (1971). Specific lymphocyte sensitization in cancer: is there a common antigen in human malignant neoplasia? Brit. med. J., 2, 613-617.

Field, E. J. and Caspary, E. A. (1970). Lymphocyte sensitization: an "in vitro" test for cancer? Lancet, 2, 1337-1341.

Goldstone, A. H., Kerr, Lorna, and Irvine, W. J. (1973). The macrophage electrophoretic migration test in cancer. Clin. exp. Immunol., 14, 469-472.

Lewkonia, R. M., Kerr, E. J. L., and Irvine, W. J. (1974). Clinical evaluation of the macrophage electrophoretic mobility test for cancer. Brit. J. Cancer, 30, 532-537.

Preece, A. W. and Light, P. Ann (1974). The macrophage electrophoretic mobility (MEM) test for malignant disease. Further clinical investigations and studies on macrophage slowing factors. Clin. exp. Immunol., 18, 543-552.

Pritchard, J. A. V., Moore, J. L., Sutherland, W. H., and Joslin, C. A. F. (1972). Macrophage-electrophoreticmobility (MEM) test for malignant disease : an independent confirmation. Lancet, 2, 627-629.

Pritchard, J. A. V., Moore, J. L., Sutherland, W. H., and Joslin, C. A. F. (1973). Evaluation and development of the macrophage electrophoretic mobility (MEM) test for malignant disease. Brit. J. Cancer, 27, 1-9. 